\title{
Biomarcadores inflamatórios e indicadores de estresse oxidativo em cadelas submetidas à ovariossalpingohisterectomia convencional, por NOTES híbrida e NOTES total
}

\author{
Inflammatory biomarkers and oxidative stress indicators in bitches subjected to \\ conventional ovarysalpingohysterectomy, by hybrid NOTES and by total NOTE
}

\author{
Paula Cristina Basso ${ }^{I^{*}}$ Alceu Gaspar Raiser ${ }^{\mathrm{I}}$ Maurício Veloso Brun ${ }^{\mathrm{I}}$ \\ Cínthia Melazzo Mazzanti ${ }^{\mathrm{I}}$ Márcio Machado Costa ${ }^{\mathrm{I}}$ Patrícia Wolkmer $^{\mathrm{I}}$ \\ Luciana Hermes Dutra ${ }^{I I}$ Daniel Curvello de Mendonça Müller ${ }^{\text {III }}$ Cássia Bagolin Silva ${ }^{\mathrm{I}}$
}

RESUMO

Objetivou-se comparar a resposta inflamatória e o perfil oxidativo da técnica de ovariossalpingohisterectomia (OSH) convencional com duas técnicas de Cirurgia Endoscópica por Orifícios Naturais (NOTES). Foram utilizados 15 fêmeas, alocadas em três grupos de cinco animais. No primeiro grupo, a OSH foi realizada por celiotomia convencional, no segundo, por NOTES total e, no terceiro, por NOTES híbrida. Foram realizadas as coletas sanguíneas antes do procedimento cirúrgico (basal), 3, 6, 12, 24, 48 e 72h pós-operatórias. A atividade da catalase mantevese alta nos três grupos estudados, entretanto a peroxidação lipídica, medida pelos níveis dos produtos de reação com o ácido tiobarbitúrico (TBARS), ocorreu mais acentuadamente no grupo convencional e foi quase que imperceptível no grupo de NOTES total. Nos três grupos estudados, ocorreu elevação na atividade da butirilcolinesterase e acetilcolinesterases, bem como aumento leucocitário neutrofílico nas primeiras horas pós-cirúrgicas. Conclui-se que a inflamação sistêmica acontece de forma similar nas três técnicas operatórias, com ressalva para as realizadas por NOTES total que mantiveram as mais baixas taxas oxidativas.

Palavras-chave:

butirilcolinesterase, acetilcolinesterase, peroxidação lipídica, enzimas antioxidantes, cirurgia sem cicatriz.

\section{ABSTRACT}

This study aimed at comparing the inflammatory response and the oxydative profile of the conventional ovarysalpingohysterectomy (OSH) technique to Totally Natural Orifice Transluminal Endoscopic Surgery (NOTES) and Hybrid NOTES. Group of fifteen female dogs was used for each technique. Blood samples were taken before the surgical procedure (basal) and 3, 6, 12, 24, 48 and $72 \mathrm{~h}$ postoperative. The catalase activity was increased in the three studied groups. Nevertheless, lipid peroxidation, measured by TBARS (thiobarbituric acid reactive substances) levels, was higher in the conventional group and almost indistinguishable in the total NOTES group. In the three analyzed groups, both butyrylcholinesterase and acetylcholinesterase activities were increased as well as the neutrophil counts during the first post-surgical hours. It is possible to conclude that systemic inflammation occurs in a similar way in the three operative techniques; however, total NOTES technique presents lower levels of cellular oxidative damage, particularly if compared to the conventional approach.

Key words: butyrylcholinesterase, acetylcholinesterase, lipid peroxidation, antioxidant enzymes, scarless surgery.

\section{INTRODUÇÃO}

A videolaparoscopia veio substituir as grandes incisões cirúrgicas, apresentando vantagens em relação à cirurgia aberta, como diminuição no tempo de internação hospitalar, menor dor pós-operatória e rápido retorno às atividades usuais. Nesse sentido, a busca por procedimentos ainda menos invasivos, aliada aos avanços da cirurgia laparoscópica e endoscópica, culminaram na Cirurgia Endoscópica Transluminal por Orifícios Naturais - NOTES (MOTTIN et al., 2007). Com essa modalidade, os cirurgiões acessam a cavidade abdominal por meio de orifícios naturais, como boca, ânus e vagina, sem envolver incisões abdominais (BARON, 2007).

\footnotetext{
'Programa de Pós-graduação em Medicina Veterinária (PPGMV), Departamento de Clínica de Pequenos Animais, Centro de Ciências Rurais (CCR), Universidade Federal de Santa Maria (UFSM), 97110-774, Rua Topázio, 100, Parque Santa Lúcia, Camobi, Santa Maria, RS, Brasil. E-mail: basso.paula@gmail.com.*Autor para correspondência.

${ }^{\text {IIC }}$ rrso de Medicina Veterinária (CMV), CCR, UFSM, Santa Maria, RS, Brasil.

IIIPrograma de Pós-graduação em Modelagem Matemática, Universidade do Noroeste do Estado do Rio Grande do Sul (UNIJUÍ), Ijuí, RS, Brasil.
} 
Segundo BAYSAL et al. (2009), o trauma cirúrgico está associado com o aumento na produção de oxidantes e diminuição dos mecanismos antioxidantes. Assim, existe a possibilidade de que procedimentos abertos estejam envolvidos com maior produção de espécies reativas de oxigênio (EROs), em virtude das grandes incisões, manipulação intestinal e ativação de células inflamatórias (SOUZA et al., 2003). Por outro lado, o pneumoperitônio, instituído nos procedimentos laparoscópicos, ocasiona redução na perfusão intestinal e na microcirculação hepática. Ao passo que a posterior desinsuflação, reduz a pressão intraabdominal, aumentando a reperfusão esplâncnica, representando um modelo de isquemia-reperfusão, fato conhecido como a principal causa de produção de radicais livres no transoperatório (BAYSAL et al., 2009). Ainda que esse modelo tenha sido estabelecido, não se tem conhecimento da produção dessas EROs no período pós-operatório, tampouco sua comparação com os procedimentos abertos.

Uma nova propriedade da acetilciolinesterase (AChE) e butirilcolinesterase (BChE) foi identificada como marcadores inflamatórios de baixo grau (DAS, 2007). Vários estudos têm demonstrado que a acetilcolina (ACh), o principal neurotransmissor do vago, tem importante ação supressora inflamatória. No entanto, a $\mathrm{ACh}$, bem como outros ésteres de colina são rapidamente hidrolisados pela AChE e BChE (MESULAM et al., 2002). Um aumento nas atividades das enzimas $\mathrm{AChE}$ e $\mathrm{BChE}$ poderia levar àdiminuição nos níveis de $\mathrm{ACh}$, reduzindo seus efeitos anti-inflamatórios, devido à ausência do controle de feedback negativo exercido pela ACh. Dessa forma, considerando o efeito inflamatório supressor da ACh, é aceitável que as atividades das enzimas AChE e BChE sejam reguladoras intrínsecas da inflamação (DAS, 2007). Sendo assim, este estudo teve como objetivo investigar os marcadores inflamatórios (AChE e BChE) e indicadores de estresse oxidativo em cadelas submetidas à OSH aberta, por NOTES híbrida e NOTES total, buscando determinar a técnica que resulta em menores alterações inflamatórias e oxidativas.

\section{MATERIAL E MÉTODOS}

Para o experimento, utilizaram-se 15 cadelas, adultas, sem raça definida, sendo alocadas em três grupos, de acordo com a técnica operatória aplicada. No primeiro grupo, a OSH foi efetuada por celiotomia retro-umbilical (GC) e, no segundo grupo, pela técnica transvaginal por NOTES híbrida (GNH). Para tanto, promoveu-se a punção da parede vaginal, junto à cérvix, para a instrumentação cirúrgica. Esse acesso foi guiado por artroscópio de
$2,7 \mathrm{~mm}$, introduzido imediatamente caudal à cicatriz umbilical. Os vasos ovarianos foram ocluídos por eletrocauterização bipolar via vaginal, enquanto os uterinos e o corpo do útero foram ligados por técnica convencional após a exteriorização dos cornos uterinos e ovários pela ferida vaginal. No terceiro grupo (GNT), aplicou-se a técnica de NOTES total, cuja sequência se assemelha em partes com a descrita para o grupo NOTES híbrida, contudo toda a instrumentalização e visualização da cavidade foram obtidas através da vagina, sem quaisquer punções para o posicionamento de portais através da musculatura abdominal. Para isso, foi necessário tracionar e incisar a mucosa do fundo vaginal, permitindo a introdução da óptica rígida com canal de trabalho. Como medicação pós-operatória, utilizouse tramadol $\left(4 \mathrm{mg} \mathrm{kg}^{-1}, \mathrm{SC}\right.$, TID) seis horas após a aplicação da medicação pré-anestésica, seguindo-se a cada 8 horas até as $72 \mathrm{~h}$ do pós-operatório.

Em todos os animais, foram realizadas coletas de sangue da veia jugular externa, nos seguintes momentos: pré-operatório, 3, 6, 12, 24, 48 e 72h pósoperatória. As amostras de sangue para hematologia foram armazenadas em tubos contendo ácidoetilenodiaminotetraacético (EDTA). Para as análises da atividade da butirilcolinesterase sérica (BChE) e dos níveis de peroxidação lipídica através das substâncias reativas ao ácido tiobarbitúrico (TBARS), $3 \mathrm{~mL}$ de sangue foram acondicionados em tubos sem anticoagulante, obtendo-se o soro por centrifugação. Para a determinaçãoda atividade da acetilcolinesterase (AChE), foi utilizado sangue total com EDTA e este foi hemolisado em $0,1 \mathrm{mmol} \mathrm{L}^{-1}$ de tampão fosfato de sódio, pH 7,4,contendo 0,03\% de Triton X-100, 1:100 $(\mathrm{v} / \mathrm{v})$. A atividade de catalase (CAT) foi determinada utilizando sangue total $(1,5 \mathrm{~mL})$ em tubos com citrato de sódio e diluída (1:10) em $\mathrm{NaCl} 0,9 \%$. As amostras para ensaio enzimático foram congeladas a $-20^{\circ}$ Caté o momento da análise.

O ensaio de AchE enzimática no sangue total foi determinado pelo método de ELLMAN et al. (1961), modificado por WOREK et al.(1999). O sistema de incubação foi composto de tampão de fosfato de sódio $0,1 \mathrm{~mol} \mathrm{~L}^{-1 \mathrm{pH} 7,4 \mathrm{e}} \mathrm{o}$ ácido 5,5'-Ditiobis2-nitrobenzoico (DTNB) 10mmol L-1, acetiltiocolina (ATC) $0.83 \mathrm{mmol}$ e adicionado hemolisado de sangue. $\mathrm{O}$ aumento da absorvância foi registrada durante 2 min a $436 \mathrm{~nm}$. Os resultados foram expressos como mU $\mu$ molde $\mathrm{Hb}^{-1}$

A atividade da BchE do soro foi determinada pelo método de ELLMAN et al. (1961). O sistema de tampão de fosfato de potássio $0,1 \mathrm{~mol}$ $\mathrm{L}^{-1}, \mathrm{pH} 7,4$, DTNB $0,30 \mathrm{mmolL}^{-1}$ e $50 \mu \mathrm{L}$ de plasma 
foi incubado durante $2 \mathrm{~min}$ a $30^{\circ} \mathrm{C}$ e a reação foi iniciada pela adição do substrato butiriltiocolina na concentração de $1 \mathrm{mmol}$. A leitura foi realizada pelo método de espectrofotometria de $2 \mathrm{~min}$ a $412 \mathrm{~nm}$. A atividade enzimática foi expressa em $\mu \mathrm{molBcSChh}^{-1} \mathrm{mg}^{-1}$ de proteína. A peroxidação lipídica foi medida no soro pelos níveis de TBARS, como descrito por JENTZSCH et al. (1996). Obtevese os resultados por meio de espectrofotometria a $535 \mathrm{~nm}$ e expressa como nanomoles de malondialdeído por mililitro de soro (nmol MDAmL-1desoro).

A determinação da atividade de CAT foi realizada pelo método modificado de NELSON \& KIESOW (1972). Este ensaio envolveu a mudança de absorvância a 240nm devido à decomposição CAT dependente de peróxido de hidrogênio. Uma alíquota $(0,02 \mathrm{~mL})$ de sangue (diluído1:10 comNaCl $0,9 \%$ ) foi homogeneizado em $0,910 \mathrm{~mL}$ de tampão de fosfato de potássio $50 \mathrm{mM}, \mathrm{pH} 7,0$. A determinação espectrofotométrica foi iniciada pela adição de $0,07 \mathrm{ml}$ de peróxido de hidrogénio $\left(\mathrm{H}_{2} \mathrm{O}_{2}\right) 0,3 \mathrm{~mol} \mathrm{~L}^{-1}$. A alteração na absorvânciaa $240 \mathrm{~nm}$ foi medida durante $2 \mathrm{~min}$.A atividade de CAT foi calculada utilizando o coeficiente de extinção molar $\left(0,0436 \mathrm{~cm}^{2}\right.$ umol $\left.^{-1}\right)$ e os resultados foram expressos como nmol de CAT por min por $\mathrm{mg}$ de proteína. As proteínas dos ensaios enzimáticos foram mensuradas pelo método de BRADFORD (1976), utilizando a albumina bovina como padrão. Para a análise estatística, utilizou-se ANOVA de uma via com posterior teste de Duncan, sendo as diferenças consideradas significativas quando $\mathrm{P} \leq 0,05$.

\section{RESULTADOS E DISCUSSÃO}

Para prevenir o dano oxidativo, o organismo dispõe de defesas enzimáticas como a superóxido dismutase (SOD), a catalase (CAT) e glutationa peroxidase (GPx), consideradas a primeira linha de defesa do organismo as EROs (FINKEL \& HOLBROOK, 2000). No entanto, em caso de produção excessiva dessas espécies reativas, como ocorre nas cirurgias, as defesas antioxidantes podem ser insuficientes em remover as EROs, evento conhecido como estresse oxidativo, iniciando assim o processo de peroxidação lipídica que pode ser danoso ao paciente (MURUGAN \& PARI, 2007). Essa peroxidação é geralmente avaliada através de TBARS, que identifica o nível malondialdeído (MDA) presente na amostra.

Em relação à atividade da CAT do grupo da cirurgia convencional, foi observado um aumento significativo na sua atividade nas primeiras $3 \mathrm{~h}$ pósoperatórias $\left(6,55 \pm 1,76 \mathrm{nmol} \mathrm{mg}^{-1}\right.$ de proteína), com ascensão contínua, até atingir seu pico máximo (Figura 1A) nas 6h pós-operatórias $(9,67 \pm 1,55 \mathrm{nmol}$ $\mathrm{mg}^{-1}$ de proteína). Esse fato demonstra a ativação de defesas antioxidantes enzimáticas, com o intuito de prevenir os danos oxidativos às membranas celulares, causados pelo trauma cirúrgico (FINKEL \& HOLBROOK, 2000). Percebe-se, entretanto, que, nas $72 \mathrm{~h}$ pós-operatórias (Figura 1A), a atividade da CAT, nesse grupo $\left(2,63 \pm 0,89 \mathrm{nmol} \mathrm{mg}^{-1}\right.$ de proteína $)$, encontra-se em níveis inferiores ao pré-operatório $\left(3,76 \pm 1,29 \mathrm{nmol} \mathrm{mg}^{-1}\right.$ de proteína), sugerindo que o processo inflamatório causado pelo trauma cirúrgico tenha sido de tamanha intensidade que possa ter ocasionado a exaustão dessa enzima. Isso é corroborado pelas observações de MURUGAN \& PARI (2007), os quais constataram que a redução da atividade das enzimas antioxidantes, como a catalase, pode ocorrer devido a sua excessiva utilização na remoção dos radicais livres, conduzindo ao esgotamento enzimático.

Nesse mesmo grupo, analisando a evolução dos níveis de TBARS no pós-operatório, verificou-se aumento significativo apenas nas $72 \mathrm{~h}$ pós-operatórias $\left(25,84 \pm 3,11 \mathrm{nmol} \mathrm{MDAmL}^{-1}\right)$, ou seja, no mesmo tempo em que coincide o possível esgotamento da enzima antioxidante (Figura 1B). Isso sugere a hipótesede que este sistema natural de defesa enzimática foi insuficiente no controle do estresse oxidativo nesse grupo, ocorrendo peroxidação lipídica, que provoca degradação da membrana e morte celular, tais como descreveram MURUGAN \& PARI (2007) e RAHIMI et al. (2005). Vale salientar que, conforme verificado na figura 1 , os níveis de TBARS foram mais elevados nos grupos que foram submetidos à cirurgia convencional com relação aos demais, sugerindo que tal procedimento esteja envolvido com danos oxidativos mais acentuados.

No que se refere à atividade da CAT do grupo NOTES total, nas primeiras $3 \mathrm{~h}$ pós-operatórias ocorreu aumento significativo na atividade enzimática $\left(7,34 \pm 0,93 \mathrm{nmol} \mathrm{mg}^{-1}\right.$ de proteína), com posterior redução significativa nas $12 \mathrm{~h}\left(2,98 \pm 0,30 \mathrm{nmol} \mathrm{mg}^{-1}\right.$ de proteína) e nas $72 \mathrm{~h}$ pós-operatórias (Figura 1C). Dessa forma, pode-se dizer que nesse grupo o sistema antioxidante foi eficaz para sequestrar os radicais livres gerados, uma vez que os níveis de TBARS reduziram significantemente nas $6 \mathrm{~h}$ pósoperatórias $\left(12,27 \pm 1,04 \mathrm{nmol} \mathrm{MDAmL}^{-1}\right)$, e foi aumentado apenas às 72 horas, momento em que os níveis foram equivalentes aos encontrados no préoperatório $\left(15,55 \pm 1,78 \mathrm{nmol} \mathrm{MDAmL}{ }^{-1}\right)$ (Figura 1D). Assim, ressalta-se que o aumento compensatório na enzima antioxidante desse grupo foi benéfico, 


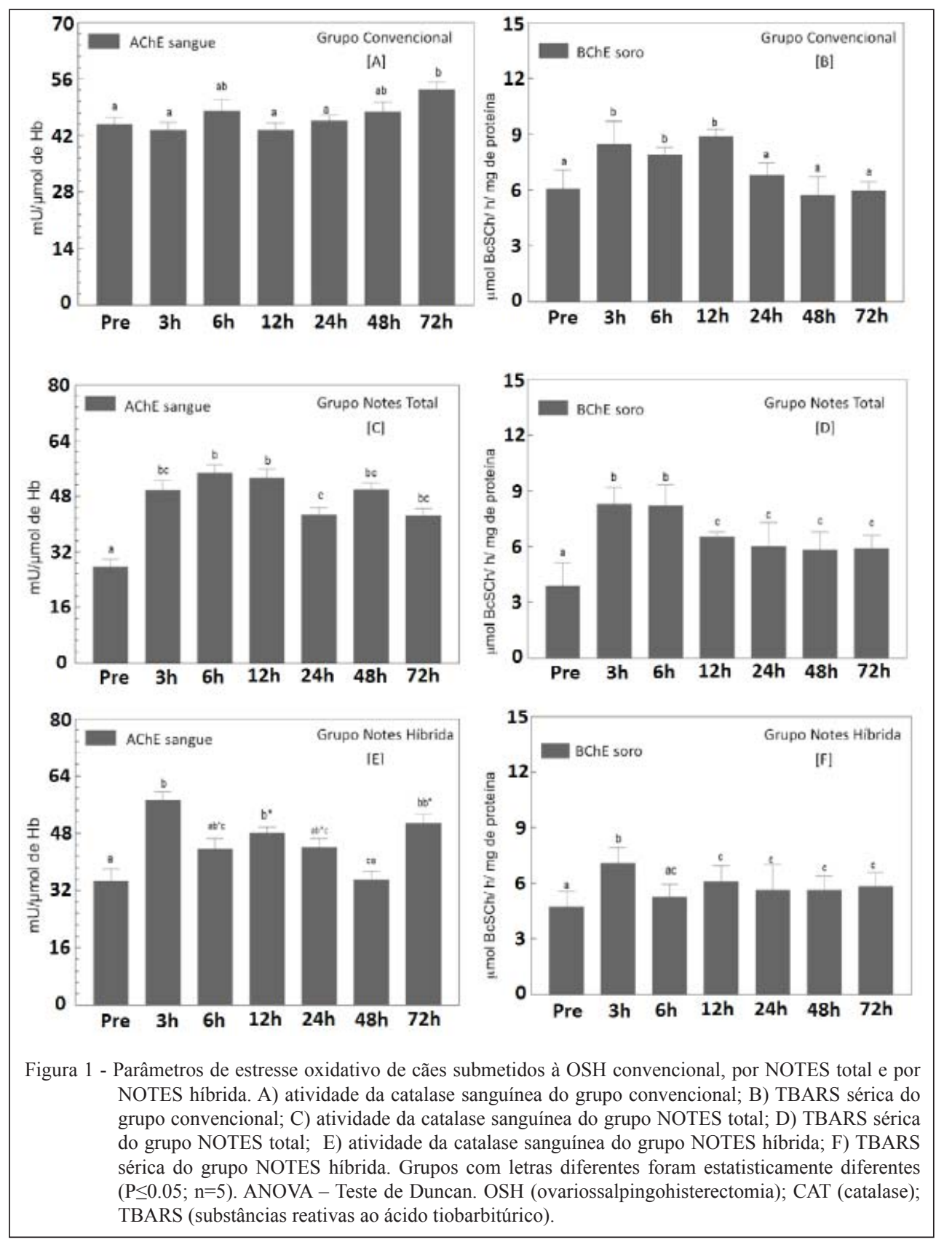

como evidenciado pelos baixos níveis de TBARS observados, o que leva a menores danos celulares no decorrer do pós operatório (TURK et al., 2002).

Por outro lado, no grupo NOTES híbrida, apesar de não ser encontrada uma elevação significativa da atividade da catalase na evolução pós-operatória, ocorreram alterações dignas de nota. Ao contrário dos demais grupos, houve redução significativa na atividade da catalase, iniciando nas primeiras $3 \mathrm{~h}\left(5,82 \pm 0,53 \mathrm{nmol} \mathrm{mg}^{-1}\right.$ de proteína) e continuando nesse mesmo sentido até as $12 \mathrm{~h}$ pós- operatórias $\left(2,64 \pm 1,32 \mathrm{nmol} \mathrm{mg}^{-1}\right.$ de proteína) (Figura 1E). Contudo, os danos na membrana plasmática aconteceram mais acentuadamente nas $12 \mathrm{~h}$ $\left(19,68 \pm 2,42 \mathrm{nmol}_{\mathrm{MDAmL}}^{-1}\right)$ e $72 \mathrm{~h}$ pós-operatórias $\left(16,38 \pm 1,96 \mathrm{nmol} \mathrm{MDAmL}^{-1}\right)$, conforme dados de TBARS (Figura 1F). Esses aumentos foram mais salientes que no grupo de NOTES total, mas não mais do que no grupo convencional. Isso sugere que o procedimento proposto de NOTES total resulta em danos oxidativos, como todo ato cirúrgico, mas que, aparentemente, paracem ser de menor intensidade 
do que os grupos operados por NOTES híbrida e por cirurgia convencional. Acredita-se que o motivo possa estar na associação do mínimo trauma tecidual ao reduzido tempo operatório (GNT: 30,42 $\pm 7,61 \mathrm{~min}$;

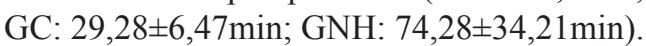

No que diz respeito à avaliação inflamatória obtida pelas dosagens de AChE sanguíneas, percebeu-se que, no grupo convencional, houve aumento significativo apenas nas $72 \mathrm{~h}$ pós-operatórias $\left(53,24 \pm 2,10 \mathrm{mU} \mu\right.$ molde $\left.\mathrm{Hb}^{-1}\right)$, demonstrando reação inflamatória mais tardia (Figura 2A). O aumento na atividade da $\mathrm{AChE}$ provavelmente ocorre para compensar a ativação da via colinérgica antiinflamatória, onde citocinas liberadas no local da infecção estimulam o núcleo dorso-motor através do nervo vago aferente. O núcleo dorso motor, juntamente com outras estruturas centrais, ativam os neurônios vagais eferentes, promovendo a liberação periférica de ACh. A ACh se liga aos receptores nicotínicos alfa7, presentes nas células do sistema imune, reduzindo e bloqueando o fator nuclear kappa $B$, inibindo assim a transcrição gênica das citocinas pró inflamatórias (DAS, 2007). Assim, com aumento da AChE, ocorre diminuição da $\mathrm{ACh}$, promovendo a resposta inflamatória nesta fase.

No entanto, no grupo de NOTES total, o aumento enzimático já ocorreu nas primeiras 6h $\left(54,47 \pm 2,50 \mathrm{mU} \mu\right.$ molde $\left.\mathrm{Hb}^{-1}\right)$, com posterior redução significativa nas $24 \mathrm{~h}$ pós-operatórias $\left(42,68 \pm 1,88 \mathrm{mU} \mu\right.$ molde $\left.\mathrm{Hb}^{-1}\right)$ (Figura $2 \mathrm{C}$ ). Este achado
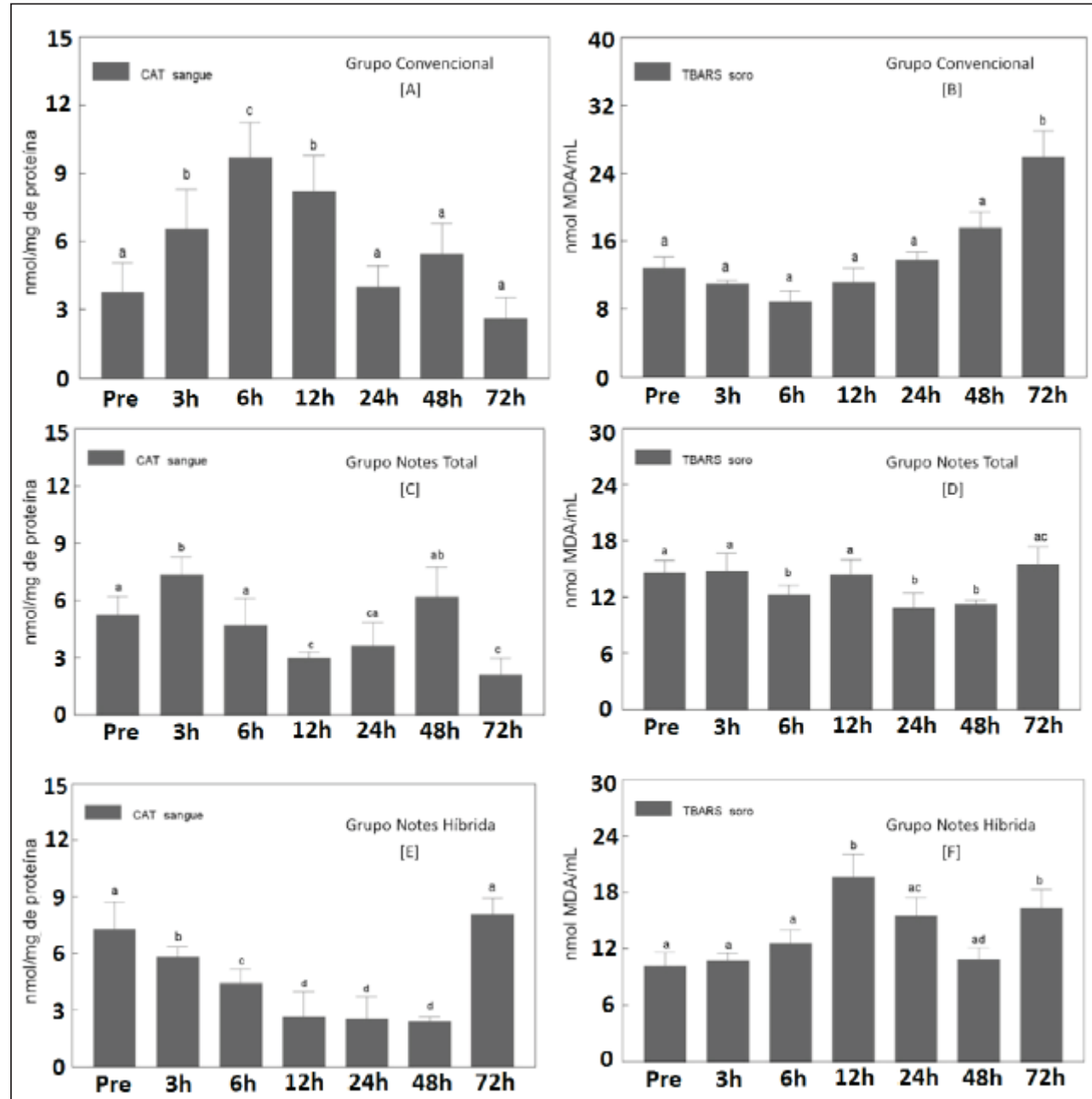

Figura 2 - Parâmetros inflamatórios de cães submetidos à OSH convencional, por NOTES total e por NOTES híbrida. A) atividade da acetilcolinesterase no grupo convencional; B) atividade da butirilcolinesterase no grupo convencional; C) atividade da acetilcolinesterase no grupo NOTES total; D) atividade da butirilcolinesterase no grupo NOTES total; E) atividade da acetilcolinesterase no grupo Notes híbrida; F) atividade da butirilcolinesterase no grupo NOTES híbrida. Grupos com letras diferentes foram estatisticamente diferentes $(\mathrm{P} \leq 0.05 ; \mathrm{n}=5)$. ANOVA - Teste de Duncan. AChE (acetilcolinesterase); BChE (butirilcolinesterase). 
está de acordo com DAS (2007), o qual afirma que a mensuração da $\mathrm{AChE}$ e BChE pode ser considerada como método confiável de avaliação da inflamação precoce. Em adição, analisando-se os dados da $\mathrm{AChE}$ no grupo de NOTES híbrida, também percebeuse a ocorrência de inflamação precoce, devido ao aumento na atividade da AChE nas primeiras 3h pósoperatórias. Esses níveis normalizaram às $6 \mathrm{~h}$ pósoperatórias, entretanto, após $12 \mathrm{~h}$ do procedimento cirúrgico, os valores aumentaram significativamente, assim como nas $72 \mathrm{~h}$ pós-operatórias (Figura 2E). $\mathrm{O}$ aumento na atividade da $\mathrm{AChE}$ em ambos os grupos NOTES já eram esperados, uma vez que a produção de pneumoperitônio transoperatório estimula alterações inflamatórias. Tal situação foi descrita por FREEMAN et al. (2010) quando compararam a dor e estresse pós-operatório de cães submetidos à ooferectomia aberta, laparoscópica e por NOTES transgástrica, com endoscópio flexível, e concluíram que, embora o procedimento por NOTES tenha evidenciado menores escores de dor, esse grupo demonstrou maior dano tecidual, quando comparado com os animais submetidos à técnica aberta, provavelmente devido ao pneumoperitônio com pressões intrabdominais mais altas e ao longo tempo cirúrgico associado à técnica proposta.

Por outrolado, analisando os valores obtidos da atividade da $\mathrm{BChE}$, percebe-se que, apesar da $\mathrm{AChE}$ ter aumentado apenas nas $72 \mathrm{~h}$ do pós-operatório no grupo da cirurgia convencional, verificou-se que a inflamação também ocorreu precocemente nesse grupo, devido ao aumento significativo da $\mathrm{BChE}$. Esse aumento ocorreu nas primeiras $3 \mathrm{~h}$ pósoperatórias $\left(8,45 \pm 1,25 \mu \mathrm{molBcSChh}^{-1 \mathrm{mg}-1}\right.$ de proteína), com diminuição até os níveis fisiológicos após $24 \mathrm{~h}$ do procedimento cirúrgico $\left(6,78 \pm 0,65 \mu \mathrm{molBcSChh}^{-1} \mathrm{mg}^{-1} \mathrm{de}\right.$ proteína) (Figura 2B). Esses dados se confrontam, de certa forma, com os da literatura, haja vista que SILVER (1974) afirma que a BChE é menos eficiente na hidrólise da $\mathrm{ACh}$ em baixas concentrações, mas altamente eficiente nas altas concentrações. Tal situação também é corroborada por LI et al. (2000), os quais relataram que a atividade da $\mathrm{BChE}$ aumenta e ocupa o lugar da AChE na degradação da acetilcolina, quando a AChE é inibida.

$\mathrm{O}$ aumento significativo da BChE também ocorreu no grupo de NOTES total nas primeiras $3 \mathrm{~h}$ $\left(8,29 \pm 0,93 \mu \mathrm{molBcSChh}^{-1} \mathrm{mg}^{-1} \mathrm{de}\right.$ proteína), vindo a reduzir significativamente nas $12 \mathrm{~h}$ pós-operatórias $\left(6,52 \pm 0,27 \mu \mathrm{molBcSChh}^{-1} \mathrm{mg}^{-1} \mathrm{de}\right.$ proteína $)$, mas não retornandoaos valores basais $\left(3,88 \pm 1,26 \mu \mathrm{molBcSChh}^{-1}\right.$ mg-1de proteína) até $72 \mathrm{~h}$ pós-operatórias (Figura 2D). Esse mesmo perfil enzimático ocorreu no grupo de NOTES híbrida, que se elevou significativamente nas $3 \mathrm{~h}$ pós-operatórias $\left(7,08 \pm 0,86 \mathrm{molBcSChh}^{-1} \mathrm{mg}^{-1} \mathrm{de}\right.$ proteína), tendendo a reduzir nas $12 \mathrm{~h}$, mas não atingindo os valores basais $\left(4,74 \pm 0,82 \mu \mathrm{molBcSChh}^{-1} \mathrm{mg}^{-1} \mathrm{de}\right.$ proteína), os quais foram alcançados apenas nas $24 \mathrm{~h}$ pós-operatórias (Figura 2F).

Destaca-se que a presença de inflamação nos três grupos testados também foi comprovada pelos dados obtidos nos hemogramas, quando ficou evidente a resposta leucocitária neutrofílica aumentada $(\mathrm{P} \leq 0,05), \quad$ caracterizando processo inflamatório agudo. Tal aumento se deu nas primeiras $3 \mathrm{~h}$ no grupo convencional $\left(13.320 \pm 260 \mathrm{~mm}^{-3}\right) \mathrm{em}$ relação aos valores basais $\left(10.320 \pm 1520 \mathrm{~mm}^{-3}\right)$, sendo observado um aumento progressivo até $12 \mathrm{~h}$ pósoperatórias $\left(19.992 \pm 1320 \mathrm{~mm}^{-3}\right)$. Porém, nos grupos NOTES híbrida e da NOTES total, elevou-se apenas a partir das primeiras $6 \mathrm{~h}$ (GNT: $15.810 \pm 960 \mathrm{~mm}^{-3}$; GNH: $16.900 \pm 1290 \mathrm{~mm}^{-3}$ ) em relação aos valores iniciais (GNT: $12.900 \pm 1430 \mathrm{~mm}^{-3}$; GNH: $\left.13.740 \pm 980 \mathrm{~mm}^{-3}\right)$. Norteado pela avaliação dos biomarcadores inflamatórios, conclui-se que todas as técnicas cirúrgicas testadas associam-se com elevado grau inflamatório, justificando o uso de anti-inflamatórios por, no mínimo, até $72 \mathrm{~h}$ pós-operatórias.

\section{CONCLUSÃO}

Conclui-se que, as três técnicas experimentais testadas conduzem à inflamação sistêmica, contudo, a técnica de NOTES total demonstra menores danos celulares, especialmente se comparados com a técnica convencional.

\section{COMITÊ DE ÉTICA}

Aprovado pelo Comitê de Ética no Uso de Animais (CEUA), registro 015/2011.

\section{AGRADECIMENTO}

Ao Conselho Nacional de Pesquisa e Desenvolvimento $(\mathrm{CNPq})$, pelo auxílio financeiro à tese, pelo processo: 300885/2008-3.

\section{REFERÊNCIAS}

BARON, T.H. Natural orifice transluminal endoscopic surgery. British Journal of Surgery, v.94, n.1, p.1-2, 2007. Disponível em: $<$ http://www.ncbi.nlm.nih.gov/pubmed/17205508>. Acesso em 03 dez. 2012.doi 10.1002/bjs.5681.

BAYSAL, Z. et al. Evaluation of total oxidative and antioxidative status in pediatric pactients undergoing laparoscopic surgery. Journal of Pediatric Surgery, v.44, p.1367-1370, 2009. Disponível em: <http://www.ncbi.nlm.nih.gov/pubmed?term=Evaluation $\% 20$ 
of $\% 20$ tota $1 \% 20$ oxidative $\% 20$ and $\% 20$ antioxidative $\% 20$ status $\% 20$ in $\% 20$ pediatric $\% 20$ pactients $\% 20$ undergoing $\% 20$ laparoscopic\%20surgery>. Acesso em: 03 dez. 2012.doi 10.1016/j. jpedsurg.2008.11.031.

BRADFORD, M.M. A rapide and sensitive method for quantification of microgram quantities of protein utilizing the principle of protein-dye binding. Analytical Biochemistry, v.72,n.1-2, p.248-254, 1976.Disponível em: <http://www.ncbi. nlm.nih.gov/pubmed/942051>. Acesso em: 07 out. 2013.

DAS, U.N. Acetylcholinesterase and butyrylcholinesterase as possible markers of low-grade systemic inflammation. Medical Science Monitor, v.13, n.12, p.214-221, 2007. Disponível em: <http://www. ncbi.nlm.nih.gov/pubmed/18049445>. Acessoem: 07 out. 2013.

ELLMAN, G.L. et al.A new and rapid colorimetric determination of acetylcholinesterase activity. Biochemical Pharmacology, v.7, p.88-95, 1961. Disponível em: <http://www.sciencedirect.com/ science/article/pii/0006295261901459>. Acesso em: 07 out. 2013.

FINKEL, T.; HOLBROOK, N.J. Oxidants, oxidative stress and the biology of aging. Nature, v.408, p.239-247, 2000. Disponível em: <http:/www.nature.com/nature/journal/ v408/n6809/full/408239a0.html>. Acesso em: 05 fev. 2012. doi:10.1033/35041687.

FREEMAN, L.J. et al. Comparison of pain and postoperative stress in dogs undergoing natural orifice transluminal endoscopic surgery, laparoscopic, and open oophorectomy. Gastrointestinal Endoscopy, v.72, n.2, p.373-380, 2010. Disponível em: <http:// www.giejournal.org/article/S0016-5107(10)00139-2/abstract>. Acesso em: 03 dez. 2012.doi: 10.1016/j.gie.2010.01.066.

JENTZSCH, A.M. et al. Improved analisys of malondialdehyde in human body fluids. Free Radical Biology \& Medicine, v.20, n.2, p.251-256, 1996. Disponível em: <http://www.sciencedirect.com/ science/article/pii/0891584995020438>. Acesso em: 07 de out. 2013.

LI, B. et al. Abundant tissue butyrylcholinesterase and its possible function in the acetylcholinesterase knockout mouse. Journal of Neurochemistry, v.75, p.1320-1331, 2000. Disponível em: <http:// onlinelibrary.wiley.com/doi/10.1046/j.1471-4159.2000.751320.x/abs tract? systemMessage $=$ Wiley + Online + Library + will + be + disrupted $+o$ $\mathrm{n}+8+$ December + from $+10 \% 3 \mathrm{~A} 00-12 \% 3 \mathrm{~A} 00+\mathrm{GMT}+\% 2805 \% 3 \mathrm{~A} 00$ -
07\%3A00+EST\%29+for+essential+maintenance>. Acesso em: 03 dez. 2012. doi:10.1046/j.1471-4159.2000.751320.

MOTTIN, C.C. et al. Colecistectomia transvaginal: relato de caso. Revista Brasileira de Videocirurgia, v.5, n.3, p.139-143, 2007. Disponível em: <http://sobracil.org.br/revista/rv050503/ rbvc050503 139.pdf>. Acesso em: 07 out. 2013.

MURUGAN, P.; PARI, L. Influence of tetrahydrocurcumin on erytrocytre membrane bound enzymes and antioxidant status in experimental type 2 diabetic rats. Journal of Ethnopharmacology, v.113, n.3, p.479-486, 2007. Disponível em: <http://www. sciencedirect.com/sciende/article/pii/S0378874107003261>. Acesso em: 04 maio, 2012. doi:10.1016/j.jep.2007.07.004.

NELSON, D.L.; KIESOW, L.A. Entalpy of the composition of hydrogen peroxide by catalase at $25^{\circ} \mathrm{C}$. Analytical Biochemistry, v.49, p.474-479, 1972.Disponível em: <http://www.ncbi.nlm.nih. gov/pubmed/?term $=$ Entalpy + of + the + composition + of + hydrogen $+p$ eroxide + by + catalase + at $+25 \% \mathrm{C} 2 \%$ BAC $>$. Acesso em: 07 out. 2013 .

RAHIMI, R. et al.A review of the role of antioxidants in the management of diabetes and its complications. Biomedicine \& Pharmacotherapy, v.59, n.7, p.365-373, 2005. Disponível em: $<$ http:// www.sciencedirect.com/science/article/pii/S0753332205001381>. Acessoem: 06 abr.2012. doi:10.1016/j.biopha.2005.07.002.

SILVER, A. The biology of cholinesterases.New York:Elsevier/ Agricultural Research Council Institute, 1974. P.426-447.

SOUZA, A.M.B. et al. Comparison of peritoneal oxidative stress during laparoscopy and laparotomy. Journal of the American Association of Gynecologic Laparoscopist, v.10, n.1, p.65-74,2003. Disponível em: <http://www.ncbi.nlm.nih.gov/ pubmed/12554997>. Acesso em: 07 out. 2013.

TURK, H.M. et al. Plasma lipid peroxidation products and antioxidants enzyme activities in patients with type 2 diabetes mellitus. Acta diabetologica, v.39, n.3, p.117-122, 2002. Disponível em: <http://link.springer.com/article/10.1007/s005920200029?noaccess $=$ true $>$. Acesso em: 07 out. 2013. doi:10.1007/s005920200029.

WOREK, F. et al. Improved determination of acetylcholinesterase activity in human whole blood. Clinica Chimica Acta, v.288, p.7390, 1999. Disponível em: <http://www.sciencedirect.com/science/ article/pii/S0009898199001448>. Acesso em: 07 out. 2013. 